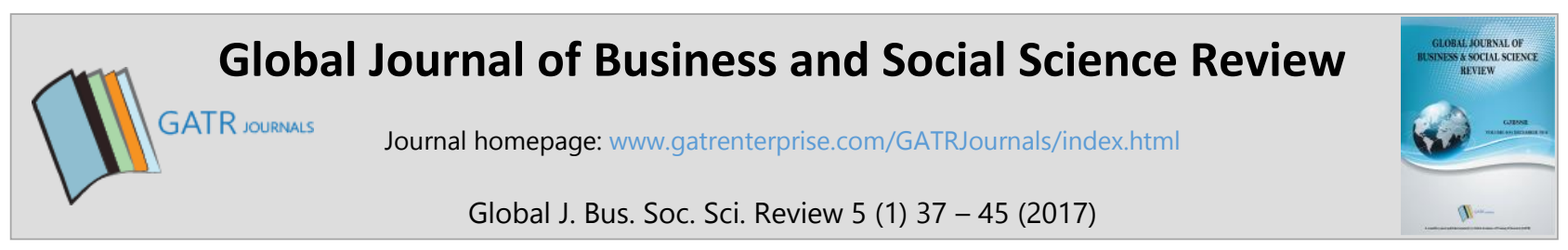

\title{
The Impact of Joint Commission International Healthcare Accreditation on Infection Control Performance: A Study in Dubai Hospital
}

\author{
Fatima Mahmoud Salim ${ }^{1}$ and Mohammad Habibur Rahman ${ }^{2 *}$ \\ ${ }^{1}$ Duabi Health Authority, PO Box 77732, Dubai, United Arab Emirates \\ ${ }^{2}$ Mohammed Bin Rashid School of Government, PO Box 72229, Dubai, United Arab Emirates
}

\begin{abstract}
Objective - The United Arab Emirates (UAE) Vision 2021 aims to achieve a world-class healthcare system and the national agenda has set multiple Key Performance Indicators (KPIs) to accomplish accreditation by all healthcare facilities in the country by 2021. The purpose of this study was to assess the impact of Joint Commission International (JCI) accreditation on Infection Control (IC) KPIs in Dubai Hospital (DH).

Methodology/Technique - A longitudinal case study covering 2007 to 2013 was conducted to examine this impact based on secondary data of the KPIs during this period and semi-structured interviews with senior health officials.

Findings - The study reveals that the healthcare accreditation acts as a catalyst for implementing and monitoring changes but it also underscores some challenges. It proposes recommendations such as improving surveillance systems, ensuring proper resources allocation, and undertaking further research.

Novelty - The study recommends improving the surveillance and reporting systems at the national level through frequent internal surveys.

Type of Paper: Empirical.
\end{abstract}

Keywords: Dubai; Infection Control; Joint Commission International Accreditation; Key Performance Indicators; United Arab Emirates.

JEL Classification: H51, I11, P46

\section{Introduction}

Improving the quality of healthcare systems will continue to gain policy attention, given the evolving expectations of people for better health management from the health service delivery institutions. Healthcare occupies a distinct place on the public policy agenda globally and global competition in the healthcare industry has been ever increasing, with many countries have been trying to attract medical tourists as a business strategy.

* Paper Info: Revised: September, 2016

Accepted: January, 2017

* Corresponding author:

E-mail: mohammad.habibur@mbrsg.ac.ae

Affiliation: Mohammed Bin Rashid School of Government, United Arab Emirates 
Having realized the importance of the healthcare sector, the UAE in its Vision 2021, incorporated an aim to achieve a world-class healthcare system (United Arab Emirates Cabinet, 2016). The UAE's National Agenda listed several national Key Performance Indicators (KPIs), of which one is achieving the accreditation by all healthcare facilities in the UAE by 2021. As part of that aim, an accreditation program with Joint Commission International (JCI), an international healthcare quality improvement agency, begun in 2007 between the JCI and Dubai Health Authority (DHA).

The JCI standards advisory panel members selected a number of best standards that are centered round the important functions common to all healthcare organizations. One of the most important chapters in the catalyst's manual of standards is the Prevention and Control of Infection (PCI) (Joint Commission International, 2014). In 1976, it became an accreditation requirement to have an Infection Control (IC) program. That requirement resulted from applying a public health model; a process of defining problems, identifying risks and protective factors, developing intervention strategies and assuring prevalent adoption and evaluation, to Healthcare Associated Infections (HAIs) (Dixon, 2011). HAIs, such as "nosocomial" and "hospital" infections, affect patients in a hospital or other healthcare facility and are not present or incubating at the time of admission (World Health Organization, 2016). HAIs were one of the top challenges facing the global healthcare sector in 2014, being the most frequent adverse event in healthcare delivery worldwide (Deloitte, 2014). These HAIs include Central Line Associated Bloodstream Infections, Catheter Associated Urinary Tract Infections, Ventilator Associated Pneumonia and Surgical Site Infections (Centers for Disease Control and Prevention, 2012).

In the UAE, Dr. Mansour Al Zarouni, chair of the Infection Control Conference and Consultant Medical and Molecular Microbiologist, Ministry of Health, UAE, reiterated that "Prevention of HAI is at the heart of patient safety" (Abu Dhabi Medical Congress, 2010). Therefore, the Dubai Health Authority (DHA), responsible for managing four hospitals (i.e. Dubai Hospital, Latifa Hospital, Rashid Hospital, and Hatta Hospital), considers the IC as one a core organizational wide functions and considers its' outcome as a significant target for improvement. In this backdrop, this study used IC as an indicator to evaluate the impact of the JCI accreditation process in Dubai Hospital (DH). It aims to assess the impact of the JCI accreditation on Infection Control KPIs in DH with the following objectives:

- to evaluate the obtained Key Performance Indicators for the period 2007-2013 in DH;

- to analyze the performance compared with the international benchmarks "control";

- to identify other factors contributing to the Infection Control; and

- to identify the impact of the JCI accreditation on IC performance in DH.

The area of this research in the context of the UAE's health sector added value to the understanding of a key health policy area and a national KPI, i.e. healthcare accreditation. Also, there is scarcity of research in the area of the impact of healthcare accreditation on the health sector in general and specifically in HAIs which have a tremendous clinical and financial impact. However, as this research is considered a single case study to answer the key research question (i.e. What is the relationship between Infection Control and JCI accreditation in Dubai Hospital?), a limitation of generalizing the findings exists. Additionally, the impact of accreditation on IC performance was looked at without considering other factors.

\section{Literature Review}

Accreditation is commonly known as "a voluntary program, sponsored by a non-governmental agencies (NGO), in which trained external peer reviewers evaluate a healthcare organization's compliance with preestablished performance standards" (Alkhenizan \& Shaw, 2012). 


\subsection{Infection control status theme}

Evidently, control of hospital acquired infections and standardization of practices is a main priority for public healthcare organizations in both developing and developed countries due to their clinical and financial implications (Emerson, et al., 2012). In 2002, the number of HAIs exceeded the number of cases of any presently notified disease, and deaths associated with HAIs in hospitals surpassed the number attributable to several of the top ten leading causes of death reported in the United States (Klevens, et al., 2007).

In 2013, a meta-analysis of costs and financial impacts of HAIs on the United States healthcare system was conducted for the period 1986 to 2013, to facilitate the process of defining the financial impact of HAIs. It was found that Central Line Associated Bloodstream Infections are the most costly HAIs, followed by Ventilator Associated Pneumonia, Surgical Site Infections, Clostridium Difficile Infection, and Catheter Associated Urinary Tract Infections. The total annual costs for the five major infections were $\$ 9.8$ billion (Zimlichman, et al., 2013).

Accordingly, a global response to the HAI problem has been lately initiated by the WHO World Alliance for Patient Safety and the project was launched as the First Global Patient Safety Challenge; "Clean Care is Safer Care". The formal statements were signed by 63 countries (Allegranzi \& Pittet, 2007).

\subsection{Healthcare accreditation positive impact theme}

The research paper prepared by the HAUTE AUTORITE DE SANTE includes an international literature review of healthcare accreditation for the period between 2000 to 2010. The review was based on 56 references. A majority of the studies/references discussed the accreditation impact as a whole without specifying a certain procedure or process. Only a few studies suggested or demonstrated a positive correlation between accreditation and improvement in the outcomes of care. Furthermore, some of the reviewed papers were based on the data of perceptions and opinions (HAUTE AUTORITE DE SANTE, 2011).

Between 2003 and 2005, an in-depth retrospective case study was carried out to determine the impact of accreditation on Canadian healthcare organizations and five Health Care Organizations with different accreditation statuses. The conclusion drawn was that the accreditation process is an effective notion for change introduction (Pomey, et al., 2010).

In 2011, a meta-analysis study on the Impact of Accreditation on the Quality of Healthcare Services was conducted, comprising 26 studies, all evaluating the accreditation impact (Alkhenizan \& Shaw, 2011). The primary conclusion was that there is consistent evidence supporting the view that accreditation programs improve the process of care. One of the reviewed papers in the meta-analysis aimed to test the impact of hospital accreditation on infection control programs in teaching hospitals in Japan. The conclusion of the study indicated that hospital accreditation had a significant impact on hospitals' IC infrastructure and performance (Sekimoto, et al., 2008). However, the research had several limitations. For example it was conducted on teaching hospitals only and the response rate was only $52 \%$.

\subsection{Healthcare accreditation antithetical theme}

There are only a limited number of studies that have been conducted which have contrary findings regarding the impact of healthcare accreditation. In 2005, a study concerning the relationship between accreditation and performance measurement was conducted to examine the association between the Joint Commission on Accreditation of Healthcare Organizations accreditation scores and the Agency for Healthcare Research and Quality's Inpatient Quality Indicators and Patient Safety Indicators (IQIs/PSIs). The findings argued that the assessment of quality from any one perspective or using any one tool was unlikely to give the complete picture (Miller, et al., 2005). Another study comparing JCI and Medicare outcome scores in hospitals concluded that JCI relies on process and structure while an outcome measure is preferred (Griffith, Knutzen, \& Alexander, 2002). 
Until today, and based on the reviewed literature, the value of accreditation remains uncertain and this continues as a legitimacy problem for accreditation providers, policy makers and researchers. Additionally, few studies have suggested or confirmed a positive correlation between healthcare accreditation and improvement in the outcomes of care. In addition, research in the developing countries are limited with several limitations and accreditation impacts on the IC performance having not been subjected to extensive study and research. Accordingly, this research assumes that there is a positive relationship between JCI healthcare accreditation and infection control KPIs in Dubai Hospital. This research intends to add value to the body of knowledge on the impact of healthcare accreditation on IC performance in the public health sector in the UAE.

\section{Research Methodology}

This research is a longitudinal case study for the period between 2007 to 2013 . This study adopted both quantitative and qualitative research methods in which data was analyzed based on an inductive approach (Pomey, et al., 2010).

The quantitative component of the study consists of the infection control KPIs data collected in Dubai Hospital between 2007 to 2013. The data was compared with benchmark standards form the Centers for Disease Control and Prevention, following their accreditation in 2010, with the aim of improving their operation.

The qualitative component of the study consists of semi-structured interviews with the Consultant of the Intensive Care Unit, Chairman of Infection Control Committee, and Head of PCI Office-DH and In-charge of the Sterile Pharmaceutical Compounding Unit, IC Committee Member-DH.

The interview questions included topics such as: infection control status before implementing the JCI accreditation standards, changes in the processes and procedures after JCI implementation, reasons for successes or failures, difficulties and challenges facing the IC Department, IC future plans, as well as the perception regarding healthcare accreditation.

\subsection{Variables}

The study has used the following variables:

Independent Variable: Joint Commission International Accreditation implementation.

Dependent Variables: Two sets of dependent variables were used to assess Infection Control performance.

The first set of variables gained from the semi-structured interviews provided further clarification about the Infection Control and healthcare accreditation in DH.

The second set of variables included the IC KPIs (Centers for Disease Control and Prevention, 2012):

- Ventilator Associated Pneumonia - VAP KPI.

- Central Line Associated Blood Stream Infection - CLABSI KPI.

- Catheter Associated Urinary Tract Infection- CAUTI KPI.

- Surgical Site Infection - SSI KPI.

\subsection{Data collection and analysis}

Collected secondary data is available in the published Dubai Health Authority (DHA) KPIs' official reports for the period between 2007 to 2013. Multivariate analysis - interrupted time series regression - was used to analyze the collected data. The SPSS20 (Statistical Package for the Social Sciences) software was used to create, modify, and analyze data ( $\mathrm{P}$ value $\leq 0.05$ was considered as the level of significance). 
The interruption (2010 JCI accreditation) separates the time series into pre-intervention and postintervention segments to evaluate the impact of the intervention on the dependent variables. In interrupted time series regression, two parameters (level and trend) were used to define each segment of the time series. Bivariate analysis (one sample t-test) was used to evaluate if the mean of test variables is significantly different from a constant (benchmark) for the period between 2011 to 2013. Regarding the semi structured interviews, a thematic analysis of the transcribed interviews was conducted.

\section{Results and Discussion}

Today, IC is one step closer toward achieving the ultimate target of patient safety. Knowing that practicing in the healthcare sector without following standards will result in many irregularities, the JCI standards have been adopted by designing policies and implementing practices to achieve standards that will be evaluated through measurable elements.

\subsection{Secondary Data Analysis}

The IC KPIs were measured on a monthly basis. In Table 1, Group 1 reflects the values for the period between January 2007 to October 2010 and Group 2 reflects the values for the period between November 2010 and December 2013. The descriptive statistics of the 4 Infection Control KPIs present a decrease in the mean after the JCI accreditation in 2010 in the Dubai Hospital. This decline can be attributed to the enhancement in the Infection Control practices.

Interrupted time series regression analysis was used to determine the significance of the differences in levels and trends over time due to the JCI accreditation. The Autoregressive Integrated Moving Average (ARIMA) model was used to evaluate the impact of the JCI accreditation in 2010 on the level and trend of the IC KPIs. The model was run in first order difference form (lagged) and the Durbin-Watson statistic test was in the inconclusive range, suggesting that there are no issues with the original model for the autocorrelation.

Table 2 presents the time series analysis for the VAP. The time series variable (measuring the trend) shows that before the intervention, there was no significant month-to-month decline in the trend. The accreditation variable (measuring level change after the intervention) shows that following the JCI accreditation in October 2010; the rate of VAP dropped significantly by 1.7.VAP and the trend was declining for the period before and after the accreditation, as the hospital started the implementation of the JCI standards and considering the measurable elements. Even though the decline in the trend after the intervention was not statistically significant, it reflects an enhancement in the processes.

Table 1. Infection Control KPIs' Descriptive Statistics

\begin{tabular}{|l|l|l|l|l|l|}
\hline $\begin{array}{l}\text { Infection } \\
\text { Control KPIs }\end{array}$ & Group & $\mathbf{N}$ & Mean & $\begin{array}{l}\text { Std. } \\
\text { Deviation }\end{array}$ & $\begin{array}{l}\text { Std. Error } \\
\text { Mean }\end{array}$ \\
\hline \multirow{2}{*}{ VAP } & 1.00 & 45 & 4.2340 & 2.47897 & .36954 \\
\cline { 2 - 6 } & 2.00 & 39 & 1.2459 & 1.12076 & .17946 \\
\hline \multirow{2}{*}{ CLA BSI } & 1.00 & 45 & 4.8320 & 2.02908 & .30248 \\
\cline { 2 - 6 } & 2.00 & 39 & 1.5562 & 1.31483 & .21054 \\
\hline \multirow{2}{*}{ CA UTI } & 1.00 & 45 & 3.5193 & 2.67501 & .39877 \\
\cline { 2 - 6 } & 2.00 & 39 & 1.9413 & 1.55033 & .24825 \\
\hline \multirow{2}{*}{ SSI } & 1.00 & 45 & .9342 & .92373 & .13770 \\
\cline { 2 - 6 } & 2.00 & 39 & .5256 & .31994 & .05123 \\
\hline
\end{tabular}


Table 2: ARIMA Model Parameters - VAP

\begin{tabular}{|l|l|l|l|l|l|l|l|l|}
\hline \multicolumn{3}{|c}{} & Estimate & SE & t & Sig. \\
\hline \multirow{3}{*}{$\begin{array}{l}\text { VAP - } \\
\text { Model_1 }\end{array}$} & Accreditation & No Transformation & Numerator & Lag 0 & -1.701 & .854 & -1.992 & .050 \\
\cline { 2 - 9 } & Time series & No Transformation & Numerator & Lag 0 & -.043 & .022 & -1.911 & .060 \\
\cline { 2 - 9 } & Time After & No Transformation & Numerator & Lag 0 & .025 & .036 & .710 & .480 \\
\hline
\end{tabular}

Table 3 presents the time series analysis for the CLABSI. The time series variable shows that before the intervention there was a significant month-to-month decline.

Table 3: ARIMA Model Parameters - CLABSI

\begin{tabular}{|l|l|l|l|l|l|l|l|l|}
\hline \multicolumn{3}{|c}{} & Estimate & SE & T & Sig. \\
\hline \multirow{3}{*}{$\begin{array}{l}\text { CLA BSI - } \\
\text { Model_1 }\end{array}$} & Accreditation & No Transformation & Numerator & Lag 0 & -1.681 & .739 & -2.273 & .026 \\
\cline { 2 - 9 } & Time series & No Transformation & Numerator & Lag 0 & -.045 & .019 & -2.305 & .024 \\
\cline { 2 - 9 } & \begin{tabular}{l} 
Accreditation After \\
\cline { 2 - 8 }
\end{tabular} & No Transformation & Numerator & Lag 0 & .014 & .031 & .450 & .654 \\
\hline
\end{tabular}

The accreditation variable shows that immediately following the accreditation in October 2010, the rate of CLABSI dropped significantly. The decline in the trend before the accreditation can be attributed to the implementation of the standards and the reporting of the identified infections.

Table 4 presents the time series analysis for the CAUTI. The time series variable shows that before the intervention there was a significant month-to-month decline. The accreditation variable shows that immediately following the accreditation in October 2010, the rate of CAUTI increased significantly. This increase in the level may be attributed to a more developed processes and surveillance after the survey, resulting in the identification of more infections. Still, the continuous trend decrease reveals proper processes and monitoring having been put in place.

Table 4: ARIMA Model Parameters - CAUTI

\begin{tabular}{|l|l|l|l|l|l|l|l|c|}
\hline \multicolumn{3}{|c}{} & Estimate & SE & t & Sig. \\
\hline \multirow{4}{*}{$\begin{array}{l}\text { CA UTI - } \\
\text { Model_1 }\end{array}$} & Accreditation & No Transformation & Numerator & Lag 0 & 1.793 & .880 & 2.037 & .045 \\
\cline { 2 - 9 } & Time series & No Transformation & Numerator & Lag 0 & -.088 & .023 & -3.825 & .000 \\
\hline
\end{tabular}

Table 5 presents the time series analysis for the SSI. The time series variable shows that before the intervention, there was a month-to-month increase in the trend. The accreditation variable shows that immediately following the accreditation in October 2010; the rate of SSI decreased by 0.50 .

Table 5: ARIMA Model Parameters - SSI

\begin{tabular}{|l|l|l|l|l|l|l|l|l|}
\hline \multicolumn{3}{|c}{} & Estimate & SE & t & Sig. \\
\hline \multirow{3}{*}{$\begin{array}{l}\text { SSI - } \\
\text { Model_1 }\end{array}$} & Accreditation & No Transformation & Numerator & Lag 0 & -.498 & .312 & -1.596 & .114 \\
\cline { 2 - 9 } & Time series & No Transformation & Numerator & Lag 0 & .010 & .008 & 1.276 & .206 \\
\cline { 2 - 9 } & $\begin{array}{l}\text { Time After } \\
\text { Accreditation }\end{array}$ & No Transformation & Numerator & Lag 0 & -.017 & .013 & -1.340 & .184 \\
\hline
\end{tabular}


Since January 2011, the three IC KPIs (VAP, CLABSI, and CAUTI) have been compared with an international benchmark. For the analysis of the significance of the data, one sample t-test was used. It is found that there is a statistically significant difference $(\mathrm{p}$ value $=0.00)$ between the variables and the benchmark.

These results showed that since 2011, the IC KPIs' mean is lower than the benchmark mean during the whole period, except for certain months. The maintained practices and KPIs point out the proper implementation of the standards and the continuous follow up to maintain the good practice. In addition, the use of the international benchmark added value in terms of not depending only on the declining trend but comparing the best practices with others.

\subsection{Interview Analysis}

a) Infection Control status before implementing the JCI standards

Before the implementation of the JCI standards, there was no IC unit, IC practices and policies or auditing unit.

b) The JCI accreditation impact on the processes and procedures

The JCI accreditation can be considered as an expert in standardizing the processes and showing the requirements that need to be implemented, "Their standards act as a road map for implementing the processes and practices". The whole system in the hospital was affected by the activation of the Infection Control setup.

c) Success/ failure aspects regarding Infection Control

The factors that contributed to the success of the infection control processes include the enforcement by leadership, policies that are monitored by all supervisors, proper allocation of budget, quick wins recognition, and mock surveys that help in assessing good practices.

d) Difficulties and challenges facing the Infection Control Department

Everlasting changes requires continuous attention. Other challenges include; resource allocation, the need for health education programs, organizational size, scarcity of infectious disease physicians, and workload. Also, professionalism in policy design is important. Organizational culture and resistance to change are another challenge. The three issues of sustainability, uncertainty, and compliance are future problems that may arise over time.

e) Infection Control future-plans

Some of the IC future plans include: utilizing technology and innovation, hiring competent staff who have been through the certification in IC, and publishing the Infection Control outcomes and reviewing the plans to be updated according to the international standards.

f) Perceptions regarding healthcare accreditation

Accreditation is similar to a test that requires preparation. Preparation is the best way to improve the practice. "Accreditation is a well-known brand to be recognized as a safe healthcare facility".

The qualitative and the quantitative approach increased the ability to interpret the findings and reveal other aspects that cannot be discovered by using only one approach (Thurmond, 2001). The quantitative data analysis revealed several key findings regarding the impact of the JCI accreditation on the IC KPIs in DH. The findings 
demonstrated a statistically significant decline in the level of VAP and CLABSI after the 2010 JCI accreditation. Furthermore, there was a significant increase in the level of CAUTI after the JCI accreditation in 2010. The level of SSI also decreased after the JCI accreditation in 2010. The trend of the three IC KPIs (VAP, CLABSI, and CAUTI) was declining in the period before and after October 2010. The SSI trend was increasing before October 2010 and started to decline after October 2010.

Additionally, comparing the IC KPIs with an international benchmark helped in realizing the improvement in the performance. The qualitative data analysis revealed that the JCI accreditation adoption led to several changes in the processes and practices of the Infection Control. The JCI accreditation provides a road map for the area of Infection Control. On the other hand, the qualitative data identified that other factors and challenges need to be considered. Those findings justify and complement the findings from the quantitative data analysis. Therefore, the study findings displayed a potentially positive impact of the independent variable (JCI accreditation) on the dependent variables (IC KPIs). With the absence of the central national disease control centre in the UAE and with the call from the National Agenda to attain the JCI accreditation, the research provides recommendations for decision makers and policy makers, in order to replicate such initiative successfully.

\section{Conclusion and Recommendations}

In conclusion, it is evident that the growth of and improvement in healthcare services have become an important area for research. Consequently, the UAE is focusing on healthcare system improvement and the call in the National Agenda reflects the government's position. In fact, the studys' quantitative and qualitative findings answer the research question by displaying a potentially positive impact of the JCI accreditation on the IC KPIs. It added to the literature especially in developing countries in terms of evaluating the accreditation impact on the Infection Control performance, which is considered a major challenge worldwide.

The study recommends improving the surveillance and reporting systems at the national level through frequent internal surveys. In addition, proper fund allocation need to be considered. Rewards and financial incentives as a kind of motivation are also important. As a future step, developing and implementing a sustainable and effective national healthcare accreditation program can be considered by the health policy makers. Finally, as with all public policies, a continuous process of policy review is important to highlight the areas that require modifications or improvements. This case study also opens the door for similar studies to be conducted regarding private healthcare facilities. Other factors that can affect the IC KPIs need to be studied and an in-depth best practice research is needed to feed in to the policy makers in the UAE and the Arab region to help them understand the benefits of international healthcare accreditation and to replicate such initiatives.

\section{References}

Abu Dhabi Medical Congress. (2010, August 16). UAE puts healthcare-associated infections under scrutiny. Retrieved April 2, 2015, from PATIENT SAFETY: http://www.patientsafety-me.com/en/Media/Past-event-press-releasesnew/Press-Release-Folder/UAE-puts-healthcare-associated-infections-under-scrutiny-/.

Alkhenizan, A., \& Shaw, C. (2011). Impact of Accreditation on the Quality of Healthcare Services: a Systematic Review of the Literature. Annals of Saudi Medicine, 407-416.

Allegranzi, B., \& Pittet, D. (2007). Healthcare-Associated Infection in Developing Countries: Simple Solutions to Meet Complex Challenges. Infection Control and Hospital Epidemiology, 1323-1327.

Centers for Disease Control and Prevention. (2012, January). National Healthcare Safety Network (NHSN) Overview. Retrieved April 2, 2015, from CDC: http://www.cdc.gov/nhsn/pdfs/validation/2012/psc-maunal-01-12-valid.pdf

Deloitte. (2014). 2014 Global health care outlook Shared challenges, shared opportunities. New York: Deloitte Touche Tohmatsu Limited.

Dixon, R. E. (2011). Control of Health-Care-Associated Infections, 1961-2011. Morbidity and Mortality Weekly Report, 58-63. 
Emerson, C. B., Eyzaguirre, L. M., Albrecht, J. S., Comer, A. C., Harris, A. D., \& Furuno, J. P. (2012). HealthcareAssociated Infection and Hospital Readmission. Infection Control and Hospital Epidemiology, 539-544.

Griffith, J. R., Knutzen, S. R., \& Alexander, J. A. (2002). Structural versus Outcomes Measures in Hospitals: A Comparison of Joint Commission and Medicare Outcomes Scores in Hospitals. Quality Management in Health Care, 29-38.

HAUTE AUTORITE DE SANTE. (2011, September). Impact and results of health care quality improvement and patient safety programmes in hospitals, What is the impact of hospital accreditation? International literature review. France.

Joint Commission International. (2014). JCI Accreditation Standards for Hospitals, 5th Edition. Illinois: Joint Commission International.

Klevens, R., Edwards, J., Richards, C., Horan, T., Pollock, D., \& Cardo, D. (2007). Estimating Health Care-Associated Infections and Deaths in U.S. Hospitals, 2002. Public Health Report, 160-166.

Miller, M. R., Pronovost, P., Donithan, M., Zeger, S., Zhan, C., Morlock, L., \& Meyer, G. S. (2005). Relationship Between Performance Measurement and Accreditation: Implications for Quality of Care and Patient Safety. American Journal of Medical Quality, 239-252.

Pomey, M. P., Charles, L. L., Champagne, F., Angus, D., Shabah, A., \& Contandriopoulos, A. P. (2010, April 26). Does accreditation stimulate change? a study of the impact of th eaccreditation process on Canadian healthcare organizations. IMPLEMENTATION SCIENCE, pp. 1-14.

Sekimoto, M., Imanaka, Y., Kobayashi, H., Okubo, T., Kizu, J., Kobuse, H., . . Yamaguchi, A. (2008). Impact of hospital accreditation on infection control programs in teaching hospitals in Japan. Am J Infect Control, 212-219. Retrieved from http://www.ncbi.nlm.nih.gov/pubmed/18371518.

Thurmond, V. A. (2001). The Point of Triangulation. Journal of Nursing Scholarship, 253-258.

United Arab Emirates Cabinet. (2016). National Agenda. Retrieved April 4, 2016, from uaecabinet: http://uaecabinet.ae/en/national-agenda

World Health Organization. (2016). The burden of health care-associated infection worldwide. Retrieved April 2, 2016 , from World Health Organization: http://www.who.int/gpsc/country_work/burden_hcai/en/\#.

Zimlichman, E., Henderson, D., Tamir, O., Franz, C., Song, P., Yamin, C. K., . . Bates, D. W. (2013). Health CareAssociated Infections A Meta-analysis of Costs and Financial Impact on the US Health Care System. JAMA Internal Medicine, 2039-2046. 